\title{
Exploring changes over time and characteristics associated with data retrieval across individual participant data meta-analyses: systematic review
}

\author{
Sarah J Nevitt, ${ }^{1}$ Anthony G Marson, ${ }^{2}$ Becky Davie, ${ }^{1}$ Sally Reynolds, ${ }^{1}$ Lisa Williams, ${ }^{1}$ Catrin Tudur Smith¹
}

1Department of Biostatistics,

University of Liverpool,

Liverpool L69 3GL, UK

2Department of Molecular and

Clinical Pharmacology,

University of Liverpool,

Liverpool, UK

Correspondence to: S J Nevitt

sjn16@liverpool.ac.uk

Additional material is published online only. To view please visit the journal online.

Cite this as: BMJ 2017;357:j1390 http://dx.doi.org/10.1136/bmj.j1390

Accepted: 18 February 2017

\begin{abstract}
OBJECTIVE

To investigate whether the success rate of retrieving individual participant data (IPD) for use in IPD meta-analyses has increased over time, and to explore the characteristics associated with IPD retrieval.
\end{abstract}

DESIGN

Systematic review of published IPD meta-analyses, supplemented by a reflection of the Cochrane Epilepsy Group's 20 years' experience of requesting IPD.

\section{DATA SOURCES}

Medline, CENTRAL, Scopus, Web of Science, CINAHL Plus, and PsycINFO.

\section{ELIGIBILITY CRITERIA FOR STUDY SELECTION \\ IPD meta-analyses of studies of all designs and all clinical areas published in English.}

\section{RESULTS}

760 IPD meta-analyses which identified studies by systematic methods that had been published between 1987 and 2015 were included. Only 188 (25\%) of these IPD meta-analyses retrieved $100 \%$ of the eligible IPD for analysis, with 324 (43\%) of these IPD metaanalyses retrieving $80 \%$ or more of relevant IPD. There is insufficient evidence to suggest that IPD retrieval rates have improved over time. IPD meta-analyses that included only randomised trials, had an authorship policy, included fewer eligible participants, and were conducted outside of the Cochrane Database of Systematic Reviews were associated with a high or complete IPD retrieval rate. There was no association

\section{WHAT IS ALREADY KNOWN ON THIS TOPIC}

Individual participant data (IPD) meta-analyses are widely regarded as the "gold standard" approach to the synthesis of data from clinical research studies but is susceptible to bias if only a proportion of IPD are available for analysis and the IPD are not representative of the patient population

IPD meta-analyses are often poorly reported in terms of proportion of IPD retrieved and reasons for non-availability of IPD

Recent years have seen a shift in attitudes and awareness towards data sharing

\section{WHAT THIS STUDY ADDS}

This systematic review of 760 IPD meta-analyses published between 1987 and 2015 showed that a quarter retrieved $100 \%$ of the eligible IPD and half retrieved less than $80 \%$ of the eligible IPD

Despite the substantial drive towards improving access to clinical research data, the IPD retrieval rate across 760 published IPD meta-analyses has not improved over time

Higher IPD retrieval rates were associated with IPD meta-analyses that only included randomised trials, had fewer eligible participants, used an authorship policy, and were conducted outside of a Cochrane review

between the source of funding of the IPD metaanalyses and IPD retrieval rate. The IPD retrieval rate of the Cochrane Epilepsy Group has declined from 83\% (up to 2005) to 65\% (between 2012 and 2015) and the reported reasons for lack of data availability have changed in recent years.

\section{CONCLUSIONS}

IPD meta-analyses are considered to be the "gold standard" for the synthesis of data from clinical research studies; however, only $25 \%$ of published IPD meta-analyses have had access to all IPD.

\section{Introduction}

Systematic reviews are considered to provide the greatest amount of evidence to support decision making in medicine. ${ }^{1}$ Individual participant data (IPD) meta-analysis is widely regarded as the "gold standard" approach to the synthesis of clinical trial data, with many documented advantages over traditional aggregate data meta-analyses..$^{2-8}$ Recent years have shown a noticeable increase in the number of published IPD meta-analyses..$^{9-11}$ An average of 49 were published each year between 2005 and 2009, ${ }^{10}$ and recent estimates suggest an increase of around four published IPD meta-analyses per year. ${ }^{11}$ IPD meta-analyses directly influence the design and conduct of clinical trials as well as clinical practice guidelines. ${ }^{1213}$

Greater resources are required to collect IPD. ${ }^{5-7}$ IPD meta-analysis is subject to the risk of selection bias and "availability bias" as only studies for which IPD are made available can be included, and these studies may not be representative of the whole evidence base. ${ }^{14} 15$ IPD meta-analyses may be delayed or abandoned owing to unclear data requesting procedures or barriers to accessing IPD. ${ }^{16-19}$ Review articles have shown that around a quarter of IPD meta-analyses published up to $2001,{ }^{20}$ up to $2005,{ }^{14}$ and even as recently as $2012^{11}$ obtained IPD for less than $80 \%$ of eligible participants. These reviews also reveal poor reporting, particularly the amount of IPD included, with between $10 \%$ and $20 \%$ of IPD meta-analyses not clearly stating how many studies and participants were eligible, were included in data requests, and were included in the analysis. ${ }^{911} 1420$ In the most recent of these reviews, only $23 \%$ of IPD meta-analyses reported the reasons for the unavailability of IPD. ${ }^{9}$

The culture of sharing clinical trial data has changed in recent years. In surveys conducted in 2011,21 22 authors of published trials reported an increased willingness to share data compared with an empirical study conducted in 2009. ${ }^{18}$ IPD sharing may be improved by the publication of data transparency strategies and 
policies by the Institute of Medicine ${ }^{23}$ and European Medicines Agency, ${ }^{24}$ a proposed policy by the International Committee of Medical Journal Editors, ${ }^{25}$ and initiatives across the wider research community as a whole. ${ }^{22} 26-28$ Indeed, the launch of data sharing initiatives such as Clinical Study Data Request (CSDR), ${ }^{29}$ a platform allowing researchers to request IPD from nearly 3000 clinical trials from 13 industry sponsors, should make access to IPD easier and faster. However, researchers have reported mixed experiences of using data sharing portals such as CSDR, suggesting that the increased safeguards may have an unintended negative impact on the conduct of IPD meta-analyses. ${ }^{30-33} \mathrm{We}$ examined whether the shift in attitudes and awareness and the increased number of options available for accessing IPD has had a positive impact on IPD meta-analyses. We systematically reviewed all published IPD meta-analyses to investigate whether the success rate of retrieving IPD for the purpose of IPD meta-analyses has increased over time, and explored the characteristics associated with IPD retrieval. We also supplemented these quantitative data by reflecting on the 20 years' experience of our research group in requesting IPD to undertake IPD meta-analyses in the specialty of epilepsy.

\section{Systematic review of IPD meta-analyses Methods}

We searched Medline, CENTRAL, Scopus, Web of Science, CINAHL Plus, and PsycINFO up to August 2015 using systematic search strategies adapted from the review by Riley et al (web appendix 1). ${ }^{10} 14$ We also consulted the reference lists from the reviews of IPD meta-analyses by Riley et $\mathrm{al}^{1014}$ (provided by the author) and Huang et $\mathrm{al}^{11}$ (available as an online appendix).

One author (SJN) screened the title, abstract, and full text of articles identified in electronic searches according to the inclusion and exclusion criteria. The principle

\section{Box 1: Characteristics of individual participant data (IPD) meta-analysis}

\section{Cochrane IPD meta-analyses}

IPD meta-analyses performed as a Cochrane review compared with non-Cochrane IPD meta-analyses

\section{Number of eligible participants}

For inclusion in IPD meta-analyses (log transformed due to skewed data)

\section{Authorship policy}

IPD meta-analyses with an authorship policy (individual authorship for those providing IPD, or collaborative group) compared with no authorship policy

\section{Inclusion of randomised studies only}

In IPD meta-analyses compared with IPD meta-analyses including non-randomised studies, diagnostic test accuracy studies, or a combination of randomised and non-randomised studies

\section{Commercial source of funding}

IPD meta-analyses with a commercial source of funding (pharmaceutical or manufacturer) compared with non-commercial sources of funding only, no funding, or no information about funding provided

\section{Age of publication}

Calculated as years before 2016 (log transformed due to skewed data) reason for exclusion was recorded for relevant articles. Any uncertainties were discussed with CTS and resolved. For accuracy, two authors (BD and SR) also screened a random sample of between 50 and 100 identified articles for eligibility; agreement between the independent screening (SJN and BD or SR) was good and any discrepancies were discussed and resolved.

\section{Inclusion and exclusion criteria}

IPD meta-analyses of studies of all types (eg, randomised, observational, diagnostic) and all clinical areas that had been published in English were eligible for inclusion. We included articles if IPD was requested from the original study investigators, if IPD was already available to review authors, or if review authors were able to extract IPD from published articles.

Methodological articles, conference abstracts, review protocols, non-clinical reviews (eg, engineering articles) were excluded. Articles including the analysis of IPD from one study as a supplement to an aggregate data meta-analysis or articles in which the primary analysis was not a synthesis (eg, prognostic model validation studies, cost effectiveness analysis) were excluded. Where duplicate publications relating to the same IPD meta-analyses were identified (eg, identical publication across multiple journals), we retained the most recently published article.

\section{Data extraction}

Information was extracted from eligible IPD meta-analyses using a piloted data extraction form (web appendix 2). The following information was extracted: year of publication, authorship policy, source of funding, clinical area, type of study, type of analysis, number of eligible studies providing IPD or aggregate data, reasons for IPD not being provided, and details of any sensitivity analyses performed to account for missing IPD.

Where published articles presented multiple IPD meta-analyses addressing different research questions with different eligible cohorts for IPD meta-analyses, we extracted information for each IPD meta-analysis. If multiple analyses were presented for the same IPD meta-analyses (eg, analysis of several outcomes), we extracted information on the maximum amount of IPD provided, even if all IPD provided were not used in IPD meta-analyses.

Information was extracted in duplicate from all eligible IPD meta-analyses. One author (SJN) extracted information from all of the eligible IPD meta-analyses and three authors (BD, SR, LW) independently extracted information from a subset of around $40 \%$ of the eligible IPD meta-analyses. Agreement between authors was good and any discrepancies were discussed and resolved.

\section{Data analysis and presentation of results}

Multivariable logistic regression was performed in Stata (version 14) to examine associations between the characteristics of IPD meta-analyses (see box 1) and a high IPD retrieval rate ( $\geq 80 \%$ compared with $<80 \%$ or unknown proportion of IPD provided) or complete IPD retrieval rate $(100 \%$ compared with $<100 \%$ or unknown 
proportion of IPD provided). (See web appendix 3 for further statistical details and several sensitivity analyses exploring the assumptions made in these analyses.)

Results of multivariable regression are presented as odds ratios and 95\% confidence intervals. Other numerical results are presented as medians and ranges or numbers and percentages as appropriate.

\section{Patient involvement}

No patients were involved in setting the research question or the outcome measures, nor were they involved in the design and implementation of the study. No patients were asked to advise on interpretation or writing up of results. There are no plans to disseminate the results of the research to study participants or the relevant patient community

\section{Results}

\section{Characteristics of IPD meta-analyses}

We identified 1278 eligible articles describing 1280 IPD meta-analyses published to August 2015 (see fig 1 and web appendix 4). IPD retrieval was not relevant for 520 of the IPD meta-analyses and therefore no further results for this subgroup of reviews are reported. These

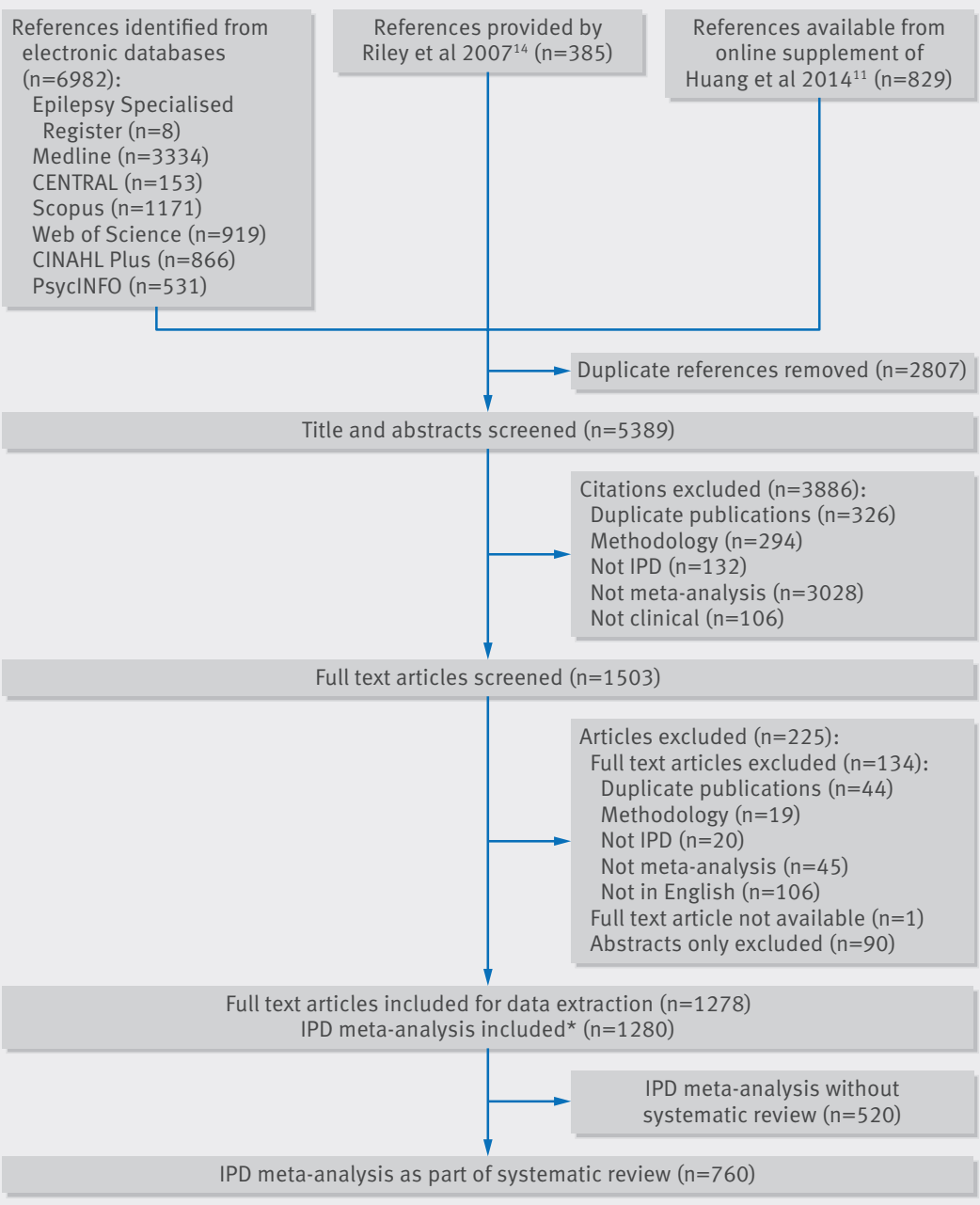

Fig 1 Study flow diagram of identification of eligible individual participant data (IPD) meta-analyses. *Two full text articles each reported two IPD meta-analyses analyses were mostly conducted with the IPD already available to analysts, by collaboration with a group of researchers who had access to the IPD, or by other non-systematic methods of identifying studies for inclusion.

For the remaining 760 IPD meta-analyses, the number of eligible studies was reported in 746 (98\%) IPD meta-analyses, with a median of 14 (range 2-923) eligible studies. The number of eligible participants within an IPD meta-analysis was reported in 510 (67\%) IPD meta-analyses, with a median of 2369 (range 16-33369) participants.

\section{IPD retrieval rate}

Out of 760 IPD meta-analyses, IPD were provided from $100 \%$ of eligible studies in only $189(25 \%)$ and from $100 \%$ of participants in only $188(25 \%)$ (table 1); one IPD meta-analysis using IPD from $100 \%$ of studies received an incomplete dataset for one study. IPD from at least $80 \%$ of eligible studies was retrieved in 375 (49\%) IPD meta-analyses and from $80 \%$ of participants in 324 (43\%) IPD meta-analyses. IPD was retrieved for less than $50 \%$ of studies in 136 (18\%) IPD meta-analyses and for less than $50 \%$ of participants in 71 (9\%) IPD meta-analyses. For 257 IPD meta-analyses, where the number of eligible participants or the number of participants excluded from IPD analysis due to lack of IPD, or both, was not reported, the proportion of IPD retrieved could not be calculated.

Figure 2 shows the number of IPD meta-analyses published by year and the proportion of IPD retrieved.

Table 1 shows the characteristics of the 760 IPD meta-analyses overall and according to IPD retrieval rate (see also web appendix figure). Among the 503 IPD meta-analyses with a known IPD retrieval rate, the odds of retrieving all IPD was statistically significantly higher in non-Cochrane IPD meta-analyses, IPD meta-analyses with a lower number of eligible participants, and IPD meta-analyses with an authorship policy (table 2). Additional analysis considering individual authorship or collaborative group authorship policies showed that the odds of retrieving $80 \%$ or more IPD were significantly higher for both types of policy, but that it was only the IPD meta-analyses offering individual authorship that were significantly associated with 100\% retrieval of IPD (see web appendix 3).

The odds of retrieving a high proportion $(\geq 80 \%)$ of IPD were also significantly higher in IPD meta-analyses of randomised trials only. There was no association between the IPD retrieval rate and source of funding, or the date of publication of IPD meta-analyses (table 2).

\section{Unavailability of IPD and impact on analysis}

Out of the 571 IPD meta-analyses that failed to retrieve IPD from 100\% of eligible studies, 201 (35\%) had supplemented IPD with aggregate data extracted from study publications. The additional aggregate data had been included from a median of 5 (range 1-541) studies and a median of 683 (range 9-1180505) participants.

At least one study had been excluded from the IPD meta-analysis owing to lack of IPD or aggregate data in 


\begin{tabular}{|c|c|c|c|c|c|}
\hline \multirow[b]{2}{*}{ IPD meta-analysis characteristic* } & \multirow{2}{*}{$\begin{array}{l}\text { Total No of IPD } \\
\text { meta-analyses }\end{array}$} & \multicolumn{4}{|c|}{ Proportion of IPD retrieved for IPD meta-analysis } \\
\hline & & $100 \%$ & $\geq 80 \%$ & $<80 \%$ & Unknownt \\
\hline \multicolumn{6}{|l|}{ Clinical area of IPD meta-analysis: } \\
\hline Breast cancer & 40 & $8(20)$ & $22(55)$ & $7(17)$ & $11(28)$ \\
\hline Cancer (other) & 53 & $14(26)$ & $27(51)$ & $14(26)$ & $12(23)$ \\
\hline Cardiology & 105 & $30(29)$ & $53(51)$ & $17(16)$ & $35(33)$ \\
\hline Central nervous system, neurology, and brain injury & 50 & $13(26)$ & $20(40)$ & $14(28)$ & $16(32)$ \\
\hline Cervical cancer and ovarian cancer & 16 & $1(6)$ & $7(44)$ & $1(6)$ & $8(50)$ \\
\hline Diabetes and endocrinology & 30 & $8(27)$ & $13(43)$ & $3(10)$ & $14(47)$ \\
\hline Gastroenterology, colorectal cancer, and gastric cancer & 49 & $11(22)$ & $17(35)$ & $23(47)$ & $9(18)$ \\
\hline Gynaecology, pregnancy, and neonatology & 35 & $13(37)$ & $18(51)$ & $9(26)$ & $8(23)$ \\
\hline Haematology, leukaemia, and blood cancer & 43 & $11(26)$ & $20(47)$ & $4(9)$ & $19(44)$ \\
\hline Head and neck cancer & 16 & $4(25)$ & $8(50)$ & $5(31)$ & $3(19)$ \\
\hline Hepatitis and liver disease & 19 & $7(37)$ & $8(42)$ & $3(16)$ & $8(42)$ \\
\hline HIV & 17 & $6(35)$ & $8(47)$ & $2(12)$ & $7(41)$ \\
\hline Infection and infectious diseases & 31 & $6(19)$ & $9(29)$ & $12(39)$ & $10(32)$ \\
\hline Injuries and wounds & 21 & $2(10)$ & $4(19)$ & $13(62)$ & $4(19)$ \\
\hline Lung cancer & 32 & $9(28)$ & $15(47)$ & $3(9)$ & $14(44)$ \\
\hline Mental and psychiatric disorders & 32 & $7(22)$ & $12(38)$ & $7(21)$ & $13(41)$ \\
\hline Musculoskeletal and pain & 34 & $9(26)$ & $11(32)$ & $5(15)$ & $18(53)$ \\
\hline Other & 26 & $5(19)$ & $9(35)$ & $12(46)$ & $5(19)$ \\
\hline Otolaryngology, ophthalmology, and periodontology & 22 & $3(14)$ & $5(23)$ & $6(27)$ & $11(50)$ \\
\hline Renal and urology & 17 & $3(18)$ & $6(35)$ & $5(30)$ & $6(35)$ \\
\hline Respiratory and pulmonary & 21 & $7(33)$ & $11(52)$ & $3(15)$ & $7(33)$ \\
\hline Stroke, thrombosis, and hypertension & 51 & $12(24)$ & $21(41)$ & $11(22)$ & $19(37)$ \\
\hline Diagnostic test accuracy & 34 & $5(15)$ & $9(26)$ & $8(24)$ & $17(50)$ \\
\hline Both randomised and non-randomised & 68 & $8(12)$ & $12(18)$ & $32(47)$ & $24(35)$ \\
\hline \multicolumn{6}{|l|}{ Type of included studies: } \\
\hline Diagnostic test accuracy & 34 & $5(15)$ & $9(26)$ & $8(24)$ & $17(50)$ \\
\hline Drug or device & 348 & $102(29)$ & $183(53)$ & $73(21)$ & $92(26)$ \\
\hline Epidemiological & 185 & $38(21)$ & $58(31)$ & $44(24)$ & $83(45)$ \\
\hline Non-drug (interventional) & 193 & $43(22)$ & $74(38)$ & $54(28)$ & $65(34)$ \\
\hline \multicolumn{6}{|l|}{ No of eligible studies: } \\
\hline $2-5$ & 102 & $72(71)$ & $83(81)$ & $10(10)$ & $9(9)$ \\
\hline $6-10$ & 174 & $67(39)$ & $98(56)$ & $34(20)$ & $42(34)$ \\
\hline $11-15$ & 120 & $16(13)$ & $47(39)$ & $27(23)$ & $46(38)$ \\
\hline $16-20$ & 87 & $12(14)$ & $29(33)$ & $27(31)$ & $31(36)$ \\
\hline $21-30$ & 101 & $6(6)$ & $30(30)$ & $28(28)$ & $43(42)$ \\
\hline $31-40$ & 50 & $3(6)$ & $11(22)$ & $19(38)$ & $20(40)$ \\
\hline $41-50$ & 29 & $2(7)$ & $5(17)$ & $9(31)$ & $15(52)$ \\
\hline$>50$ & 83 & $10(12)$ & $19(23)$ & $24(29)$ & $40(48)$ \\
\hline Not stated & 14 & $0(0)$ & $2(14)$ & $1(7)$ & $11(79)$ \\
\hline \multicolumn{6}{|l|}{ No of eligible participants: } \\
\hline$\leq 100$ & 18 & $14(78)$ & $16(94)$ & $1(6)$ & $0(0)$ \\
\hline $101-200$ & 20 & $13(65)$ & $16(80)$ & $4(20)$ & $0(0)$ \\
\hline $201-500$ & 45 & $21(47)$ & $25(56)$ & $19(42)$ & $1(2)$ \\
\hline $501-1000$ & 67 & $35(52)$ & $45(67)$ & $22(33)$ & $0(0)$ \\
\hline $1001-5000$ & 198 & $70(35)$ & $134(68)$ & $61(31)$ & $3(1)$ \\
\hline $5001-10000$ & 62 & $13(21)$ & $37(60)$ & $23(37)$ & $2(3)$ \\
\hline$>10000$ & 100 & $22(22)$ & $53(53)$ & $46(46)$ & $1(1)$ \\
\hline Not stated & 250 & $0(0)$ & $0(0)$ & $0(0)$ & $250(100)$ \\
\hline \multicolumn{6}{|l|}{ Type of IPD meta-analysis: } \\
\hline Cochrane review & 64 & $10(16)$ & $25(39)$ & $27(42)$ & $12(19)$ \\
\hline Non-Cochrane review & 696 & $178(26)$ & $299(43)$ & $152(22)$ & $245(35)$ \\
\hline \multicolumn{6}{|l|}{ Authorship policy: } \\
\hline Individual authorship & 243 & $84(35)$ & $116(48)$ & $39(16)$ & $88(36)$ \\
\hline Collaborative group & 264 & $40(15)$ & $119(45)$ & $43(16)$ & $102(39)$ \\
\hline None & 253 & $64(25)$ & $89(35)$ & $97(39)$ & $67(26)$ \\
\hline \multicolumn{6}{|l|}{ Design of included studies: } \\
\hline Randomised & 405 & $117(29)$ & $222(55)$ & $83(20)$ & $100(25)$ \\
\hline Non-randomised & 253 & $58(23)$ & $81(32)$ & $56(22)$ & $116(46)$ \\
\hline
\end{tabular}


Table 1 | Characteristics of all individual participant data (IPD) meta-analyses according to proportion of IPD provided. Values are numbers (percentages) unless stated otherwise

\begin{tabular}{|c|c|c|c|c|c|}
\hline \multirow[b]{2}{*}{ IPD meta-analysis characteristic* } & \multirow{2}{*}{$\begin{array}{l}\text { Total No of IPD } \\
\text { meta-analyses }\end{array}$} & \multicolumn{4}{|c|}{ Proportion of IPD retrieved for IPD meta-analysis } \\
\hline & & $100 \%$ & $\geq 80 \%$ & $<80 \%$ & Unknownt \\
\hline \multicolumn{6}{|l|}{ Source of funding: } \\
\hline Commercial & 72 & $26(36)$ & $37(51)$ & $14(20)$ & $21(29)$ \\
\hline Mixed & 35 & $8(23)$ & $20(57)$ & $7(20)$ & $8(23)$ \\
\hline Total & 760 & $188(25)$ & $324(43)$ & $179(24)$ & $257(34)$ \\
\hline
\end{tabular}

419 (55\%) IPD meta-analyses. Across these, a median of 4 (range 1-342) studies and a median of 478 (range 8-1792339) participants were excluded from IPD meta-analyses, but 241 (32\%) IPD meta-analyses failed to state how many participants were excluded from analysis.

Up to six reasons were reported for unavailability of IPD (table 3) for each meta-analysis; unspecific reasons, such as data not available, were reported in $341(60 \%)$ out of 571 IPD meta-analyses. The most common specific reasons for not obtaining IPD were that investigators could not be contacted, investigators had declined to share data, or data had been lost or destroyed. In 24 IPD meta-analyses it was reported that data were not requested for all studies; mainly owing to the size or quality of these studies.

In $143(25 \%)$ of the 571 IPD meta-analyses there was no acknowledgment of potential bias resulting from missing IPD. In 199 (34\%) of the IPD meta-analyses additional analyses using aggregate data had been

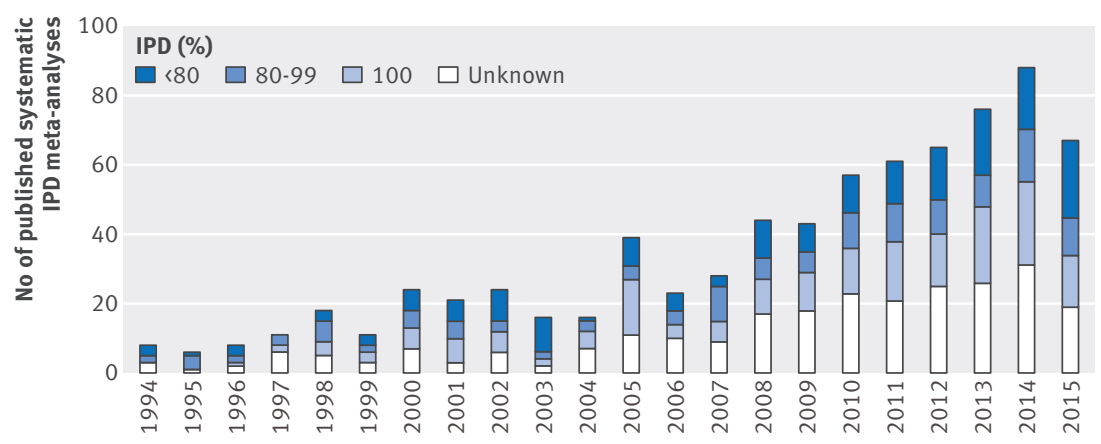

IPD (\%)

$\begin{array}{llllllllllllllllllllll}38 & 17 & 38 & 0 & 17 & 27 & 25 & 29 & 38 & 63 & 6 & 21 & 22 & 11 & 25 & 19 & 19 & 20 & 23 & 25 & 20 & 33\end{array}$ $\begin{array}{llllllllllllllllllllllllll}80-99 & 25 & 67 & 25 & 27 & 33 & 18 & 21 & 24 & 13 & 13 & 19 & 10 & 17 & 36 & 14 & 14 & 18 & 18 & 15 & 12 & 17 & 16\end{array}$ $\begin{array}{lllllllllllllllllllllll}100 & 0 & 0 & 13 & 18 & 22 & 27 & 25 & 33 & 25 & 13 & 31 & 41 & 17 & 21 & 23 & 26 & 23 & 28 & 23 & 29 & 27 & 22\end{array}$

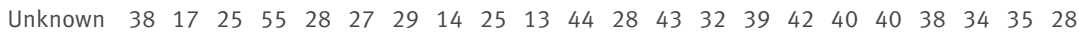

$\begin{array}{lllllllllllllllllllllll}\text { Total IPD } & 8 & 6 & 8 & 11 & 18 & 11 & 24 & 21 & 24 & 16 & 16 & 39 & 23 & 28 & 44 & 43 & 57 & 57 & 65 & 76 & 88 & 67\end{array}$ meta-

analyses

Fig 2 | Number of distinct systematic individual participant data (IPD) meta-analyses published to August 2015 and proportion of IPD provided. See table 1 for proportion of IPD meta-analyses providing $100 \%, 80-99 \%$, and less than $80 \%$ of IPD and the proportion of IPD not reported. Six IPD meta-analyses were published from 1987 to 1993; one was provided with less than $80 \%$ of IPD, three with $80-99 \%$ of IPD, and for two the proportion of IPD provided was not reported performed and in a further $66(11 \%)$ a narrative description of the studies without IPD or a narrative comparison with an aggregate data meta-analysis had been provided (table 4). The remaining 183 (31\%) IPD meta-analyses make reference to the missing data; some acknowledging that this may result in bias, without any further investigation of the implication on the conclusions of the review.

\section{Changes in data sharing over time in epilepsy}

The Cochrane Epilepsy Group has been requesting IPD from authors of trials of antiepileptic drug monotherapy since the mid-1990s. Eight reviews for IPD meta-analyses of pair wise antiepileptic drug comparisons have been published since 2000. ${ }^{34-41}$

It is believed that with effective antiepileptic drug treatment, at least $70 \%$ of people with active epilepsy have the potential to become seizure-free and go into long term remission shortly after starting treatment with one antiepileptic drug. More than 50 drugs are available worldwide for the treatment of epileptic seizures. The correct choice of first line drug for those with newly diagnosed epilepsy is of great importance, and evidence for the relative effectiveness and tolerability of antiepileptic drugs appropriate to given seizure types should be considered. ${ }^{42}$

IPD is particularly desirable for meta-analysis of trials on antiepileptic drugs to allow complete reanalysis of important time to event outcomes such as time to withdrawal of randomised treatment owing to poor seizure control or adverse effects, the recommended primary effectiveness outcome of drug monotherapy trials, ${ }^{43}$ and to allow investigation of interaction between treatment and epilepsy type, as well as other potential prognostic factors of interest. ${ }^{44}$ The group has also published an IPD network meta-analysis including participants randomised to one of eight antiepileptic drugs in the earlier phase of reviews. ${ }^{45}$ This network meta-analysis is now currently being expanded as a full Cochrane review of 10 antiepileptic drugs. ${ }^{42}$

Web appendix 5 shows IPD retrieval rates and reasons given to us for the unavailability of IPD (where applicable) categorised by the year in which requests for IPD were initiated and according to the type of study sponsorship (industry, government, and academic studies). Academic studies were defined as those conducted 


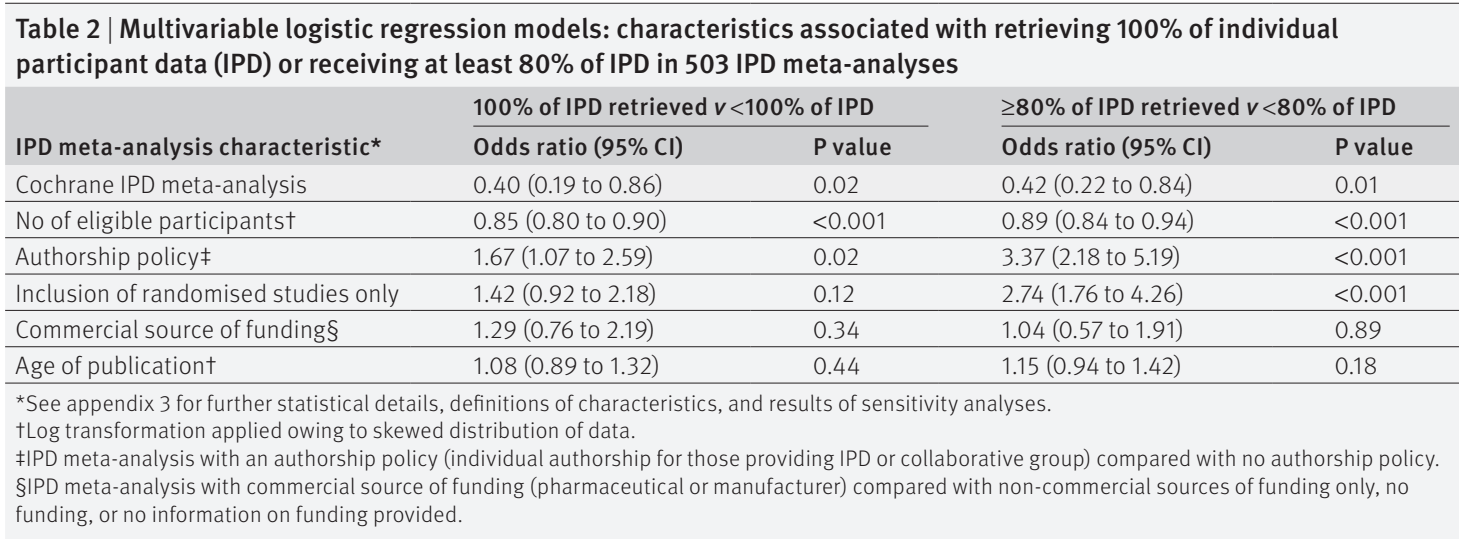

within a university or hospital setting without clear industry or government sponsorship or involvement.

Early data requesting and data sharing experiences For the reviews and network meta-analyses published up to $2007,{ }^{34} 45-51$ we requested IPD for a total of 5887 participants from 29 randomised trials and we successfully received IPD for 4703 (80\%) participants from 18 (62\%) of these eligible trials. In addition, we had IPD available from our own SANAD trial, 5253 the largest trial in epilepsy at the time, in which 2437 participants were randomised. More than $90 \%$ of IPD requested from industry and government sponsored studies were successfully received (data provided for 3695 out of 4084 participants from 12 (86\%) out of 14 studies). Only 56\% of IPD requests from academic studies were successfully received (data provided for 1008 out of 1803 participants from 6 (40\%) out of 15 studies) (web appendix 5).

We failed to retrieve IPD from 11 (38\%) eligible trials recruiting 1184 participants. For most of these trials, data had been lost or was no longer available due to the elapsed time (web appendix 5).

Many of the data requests were initiated at a time (in 1990s) when IPD meta-analyses designs were relatively novel and when email was not commonly used. Requests to trial investigators were made by letter, fax,

Table 3 | Reasons reported for unavailability of individual participant data (IPD) in 571 IPD meta-analyses without $100 \%$ of IPD (study level) retrieved

\begin{tabular}{ll} 
Reasons reported for not retrieving $\mathbf{1 0 0} \%$ of eligible IPD & No (\%) of IPD meta-analysis* \\
Data not availablet & $341(60)$ \\
\hline Investigators could not be contacted & $104(18)$ \\
\hline Investigators declined to share data & $74(13)$ \\
\hline Data lost or destroyed & $65(11)$ \\
\hline Data could not be extracted $\neq$ & $55(10)$ \\
\hline Trial was still ongoing & $42(7)$ \\
\hline Data quality issues & $29(5)$ \\
\hline Failed to provide data in time for IPD meta-analyses & $26(5)$ \\
\hline Data not requested & $24(4)$ \\
\hline Ethical or ownership restrictions & $15(3)$ \\
\hline Reason unclear & $11(2)$ \\
\hline
\end{tabular}

*189 IPD meta-analyses with $100 \%$ of IPD provided not included in table. IPD meta-analyses reported up to six reasons for unavailability of IPD. Therefore total number of reasons is greater than 571 and total percentages sum to $>100$.

tReason "data not available" corresponds to a statement in the review that IPD were not available for a proportion of studies, without any specific reason given.

¥Reason applicable only in a small number of IPD meta-analyses where IPD were extracted from publications rather than requested. telephone, and in person. Some datasets supplied had never been computerised. Because of the informal nature of many of these requests, no data sharing agreements were exchanged and little documentation was retained about the response time to data requests. Therefore, we are unable to make formal numerical comparisons between early and recent data requests; all comparisons are anecdotal.

\section{Recent data requesting and data sharing experiences}

Since our original network meta-analysis, additional antiepileptic drugs have been used in clinical practice and additional clinical trials have been conducted which has prompted the need to update our original network meta-analysis. We carried out a new search for clinical trials and this identified 39 further eligible trials to be included with the previously received IPD (total of 68 trials). ${ }^{42}$ Requests for IPD for the additional eligible studies began in January 2012 and the database was closed at the end of 2015 to begin analysis (fig 3). In total, IPD for 8261 participants from 39 additional trials were requested. Four of the requests for industry studies were made through ClinicalStudyDataRequest.com (CSDR) (known as GSK Share between May 2013 and January 2014). All other requests were made directly to the relevant sponsor.

For each trial meeting our inclusion criteria, we sent a data request to the first or corresponding author of the trial, or both, or to the trial sponsor, as appropriate. Requests were sent by as many methods as possible (email, post, fax). In the event of no response, we sent a follow-up communication to the author or sponsor previously contacted. If we still received no response, we attempted to contact another trial author or sponsor where possible.

At the close of the database at the end of December 2015, IPD had been received for 5335 (65\%) participants from 15 (38\%) clinical trials (web appendix 5). For these trials, the median time from initial request to receiving a response was similar for the 24 academic studies (343 days (range 15-861 days)) and the 14 industrial studies (363 days (range 17-725 days)) The time taken to receive IPD for one trial (study 27) using CSDR was 364 days. We note that the request was first submitted in June 2013 when the platform was newly initiated and processes 


\begin{tabular}{|c|c|}
\hline Approach reported to account for missing IPD & No of IPD meta-analyses* \\
\hline None stated & $143(25)$ \\
\hline Additional analyses performed using aggregate data: & $199(34)$ \\
\hline Separate meta-analyses are conducted including IPD only and IPD plus available aggregate data & $81(14)$ \\
\hline Aggregate data included in primary analysis & $61(11)$ \\
\hline Sensitivity analysis with aggregate data performed & $57(10)$ \\
\hline Narrative description of studies without IPD or narrative comparison with aggregate data meta-analysis had been provided: & $66(11)$ \\
\hline Results from studies without IPD summarised narratively & $48(8)$ \\
\hline Narrative comparison with aggregate data meta-analysis & $18(3)$ \\
\hline Stated that missing IPD are a limitation of the meta-analysis or that availability bias might be present, or both & $76(13)$ \\
\hline Stated that missing IPD are unlikely to change results & $56(10)$ \\
\hline Stated that majority of data are included in analysis & $47(8)$ \\
\hline Intend to include data in an update & $14(2)$ \\
\hline
\end{tabular}

were still under development. Current response times from CSDR may differ.

We failed to retrieve IPD from 24 trials conducted between 1989 and 2012. We were provided with a reason in 11 trials that had recruited 1537 participants; the median time from initial request to negative response from these 11 studies was 287 days (range 0-764 days).

Reasons for negative response were: (a) restrictions specific to a country over anonymisation of data (one request submitted to CSDR for an industry study conducted in 2005), (b) cost of retrieving and preparing data was prohibitive owing to the age of the study (two requests submitted to CSDR for industry studies conducted in 2002 and 2007), (c) data could not be made available, no more specific details were provided (three requests directly to industry for studies conducted between 1997 and 2007), (d) concerns about ethical approval for sharing data (one academic author, study conducted 2011), (e) the data we requested had not been recorded (one academic author, study conducted 2005), and (f) data were lost (three academic authors of studies conducted between 1992 and 2012; one of which provided additional unpublished summary data).

For the remaining 13 trials, two (one government sponsored and one academic) had indicated an initial positive response to our IPD requests, but data were not provided by the close of database, whereas 11 studies (nine academic and two industry sponsored) gave no response. The 13 data requests were closed at a median of 972 days (range 640-1448 days) after initial request (fig 3, web appendix 5).

Therefore at the close of the database, the total number of IPD provided for network meta-analysis was $10038(71 \%)$ of 14148 eligible participants from 33 (49\%) of 68 eligible studies (the initial 29 studies supplemented with the 39 studies described in this article).

\section{Discussion}

Recent years have shown an increase in development of statistical methods for the synthesis of individual participant data (IPD) ${ }^{54}$ as well as a rapid increase in the uptake of methods, with the number of systematic and non-systematic IPD meta-analyses published each year increasing to an average of 105 yearly between 2009 and 2015 compared with 49 yearly between 2005 and 2009.10 However, these rapid increases do not seem to be mirrored by improved IPD retrieval rates, which may be partly owing to the increasing uptake of IPD meta-analyses across a wide range of clinical areas and settings where it might be difficult to obtain IPD.

The first in the series of Cochrane Epilepsy Group IPD meta-analyses was published in 2000 when such an approach was relatively new and methods were limited. ${ }^{34}$ This meta-analysis included IPD from $63 \%$ of total studies and $83 \%$ of total participants, a good retrieval rate in the wider context of all IPD meta-analyses. The success rate has declined from more than 80\% (up to 2005) to 65\% (between 2012 and 2015), which is a concern. The findings of our systematic review showed that all or a high proportion of IPD from Cochrane reviews were less likely to be retrieved than for non-Cochrane reviews. This might be explained by the inclusion of thorough search methods within Cochrane reviews, as well as advances in systematic searching of larger electronic databases generally, leading to the identification of larger numbers of studies, including more grey literature studies where IPD may be difficult to retrieve with the resources available to review authors, such as Cochrane review authors who usually undertake systematic reviews on a voluntary basis.

Also of concern are changes in the reported reasons for data not being available. Our results show that loss of datasets is a problem for academic trials and has been for many years, highlighting a need for better methods of data collection and archiving. In our more recent requests, the prohibitive costs have prevented the sharing of pharmaceutical data. Additional costs and resources associated with IPD meta-analyses are generally considered to be incurred by the meta-analysts $^{5-7}$; however, in this new era of commercial data sharing platforms ${ }^{29}$ and requirement for high level anonymisation of data, costs to data providers are likely to have increased and should be considered when planning an IPD meta-analysis. Collaboration, financial or otherwise, between meta-analysts and data providers 


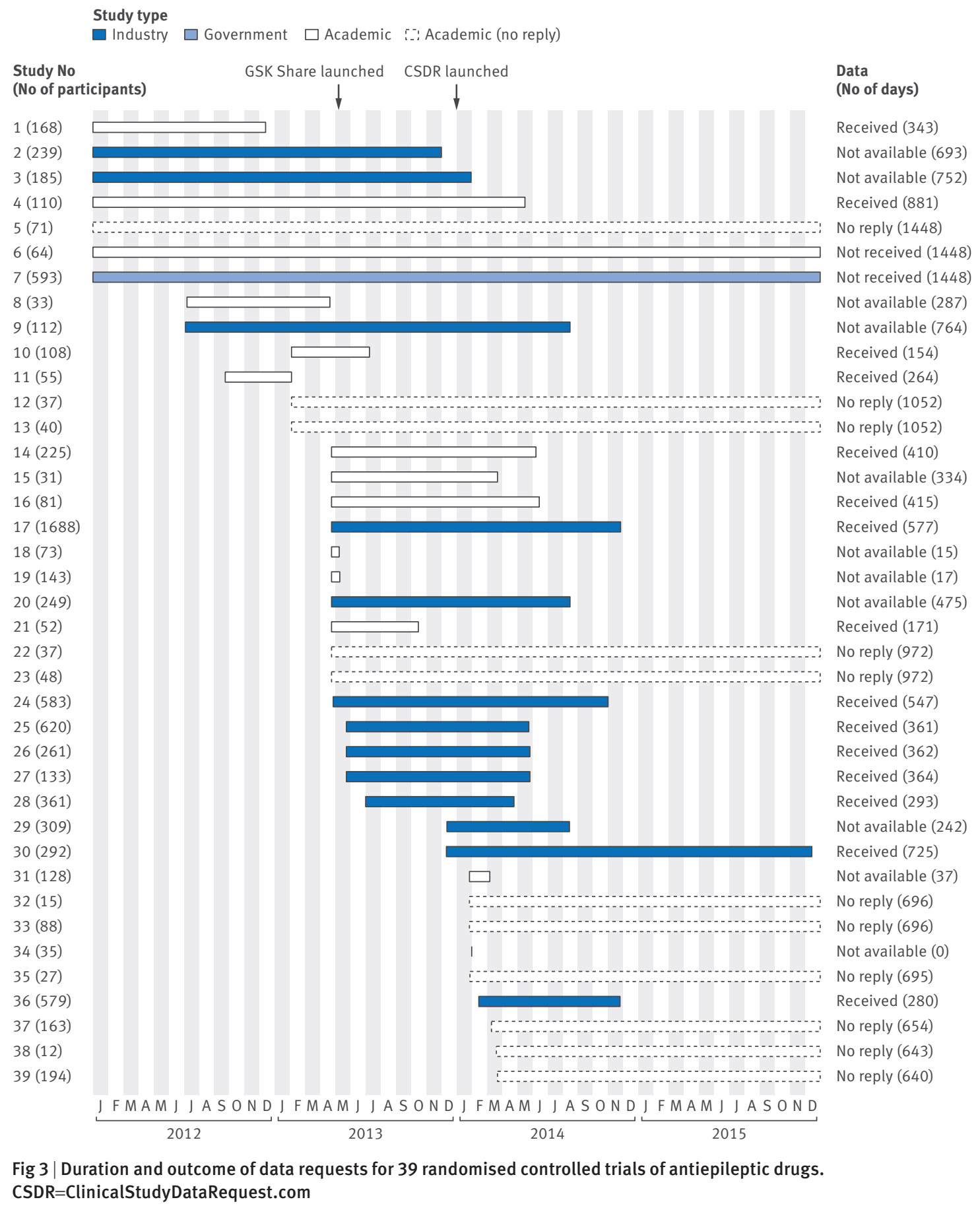

may assist in sharing costs and resources, potentially maximising retrieval rates of IPD.

The findings of our systematic review also showed that IPD meta-analyses with an authorship policy, ideally concerning individual authorship, were associated with a high or complete IPD retrieval rate. This is an important finding as the implementation of an authorship policy as an incentive to participate in an IPD meta-analysis, as a feature of a well designed project, is a factor which is in control of the IPD meta-analysis team; even where other characteristics such as study design and number of eligible participants for IPD meta-analyses are constrained by the research question.
Despite our highlighted concerns, recent changes in methods of data sharing have resulted in several benefits to our analyses. Our most common reason for not being able to retrieve data for academic trials was because we failed to make contact with data providers. In our experience, facilities within industry data sharing platforms allowed a clear and transparent pathway of communication between data requestors and providers. The continued benefit of such facilities will require increasing providers, from industry, government, and academia.

In addition to improvements to good clinical practice guidelines, developed jointly with regulations such as uptake of such platforms from both data users and data 
the European Union Clinical Trials Directive, ${ }^{55}$ a greater focus on data privacy and additional preparation required to share a dataset has resulted in cleaner datasets provided to us in recent requests compared with previous requests. While under the new framework of data sharing platforms, additional time and resources must allow for constructing a research proposal, independent scientific review, signing of data sharing agreements, and anonymisation of data. Recent datasets provided to us have required much less data cleaning before analysis than in previous years, which has led to a reduction in the time required to perform an IPD meta-analysis.

\section{Strengths and weaknesses of this study}

To the best of our knowledge at the time of writing, our systematic review includes the largest cohort of published IPD meta-analyses to date. We aimed to systematically identify all published IPD meta-analyses regardless of use of a systematic design to identify studies, resulting in a large cohort of nearly 1300 IPD meta-analyses. Our inclusion criteria were wide and reasons for exclusion were documented for all references identified in electronic searches. We were unable to include 90 abstracts as they could not be matched to full text articles, despite our best efforts. Because of the size of the cohort of this study, double reference screening and data extraction were performed on only a subset of the articles. Agreement was good and all discrepancies were minor and easily resolved; therefore we believe that any errors during screening and extraction would be minimal and unlikely to influence the overall findings of the study.

We were unable to systematically investigate the IPD retrieval methods employed within the IPD meta-analyses; for example, number of attempts to contact investigators to request data, owing to the lack of published detail on such processes. Data collection methods are likely to impact on the proportion of IPD provided for analysis, and the clearer reporting of approaches to IPD collection, such as the approach of our research group that we have outlined, may prove valuable to those planning new IPD meta-analyses.

Within our primary analysis, we performed multivariable logistic regression analysis on $100 \%$ of IPD retrieved and $80 \%$ or more of IPD retrieved. We note the limitation of dichotomisation; however, we believe that any loss of information is reduced by the size of the cohort included in analysis, and we have performed a range of sensitivity analyses to investigate all assumptions we have made in our primary analysis, showing consistency and robustness in our results (see web appendix 3 for rationale and numerical results of all sensitivity analyses performed).

We emphasise when interpreting the timelines of our requests between 2012 and 2015, that data sharing policies and platforms were under development, and that all of the industrial sponsors we contacted directly at the time of request have since committed to CSDR or an equivalent data sharing platform such as YODA (Yale University Open Data Access). .5

\section{Relation to other studies and implications}

Our results show that a quarter of IPD meta-analyses published since 1987 retrieved all IPD for analysis and only half retrieved at least $80 \%$ of relevant IPD. This latter finding is higher than previous results, which reported that around 25\% of IPD meta-analyses had included less than 80\% of IPD. ${ }^{911} 1420$ However, previous work has been based on smaller cohorts of IPD meta-analyses, mostly focused on IPD meta-analyses of randomised controlled trials only, and been conducted over shorter time frames.

In line with previous work, 9111420 our results show that important inadequacies in the conduct and reporting of IPD meta-analyses remain. Non-systematic methods, mostly based on the known availability of IPD, had been used to select eligible studies for inclusion in $41 \%$ of the initial cohort of IPD meta-analyses that we identified. It was outside the scope of this study to further examine the design of these analyses; however, we recommend that non-systematic pooling of IPD is conducted in the framework of a prospective meta-analysis and that the conclusion of such analyses must be made, taking into account the inevitable selection bias. ${ }^{56}$

Our results also highlight the importance of clear reporting of the study and participant numbers contributing to different stages of the IPD meta-analysis, with an adequate investigation of the reasons for lack of data and discussion of the potential for data availability bias. The total number of eligible participants and the total number of participants' data requested was unclear in 34\% of published IPD meta-analyses; in 58\% of the IPD meta-analyses that failed to retrieve $100 \%$ of eligible IPD, no specific reasons were provided for the unavailability of data, making interpretation of IPD meta-analyses results and conclusions in the presence of potential availability bias difficult; and in a quarter of IPD meta-analyses unable to retrieve $100 \%$ of IPD, there was a complete lack of discussion or acknowledgment of availability bias. A systematic investigation of the impact of availability bias on the conclusions of IPD meta-analyses was outside the scope of this review and is likely specific to the clinical context in question. Furthermore, our own experiences of requesting IPD show that this issue is not restricted to the reporting of IPD meta-analyses but that it also exists at the study request level; IPD from three out of 35 studies were not available to us, with no further reason stated (web appendix 5). Despite this, further efforts are recommended by researchers conducting an IPD meta-analysis to thoroughly investigate and report the impact of data availability. ${ }^{15}$

We hope that the uptake of PRISMA (Preferred Reporting Items for Systematic Reviews and Meta-Analyses) guidelines for the conduct and reporting of IPD meta-analyses, ${ }^{57}$ in addition to guidance on the use of IPD meta-analysis to synthesise the results of randomised controlled trials, ${ }^{58}$ will lead to improved conduct and reporting in IPD meta-analyses, particularly regarding transparent reporting of the number of eligible studies and participants, how much data were requested and obtained, with clear reasons for 
non-availability of IPD, preferably with a flow diagram, and data collection methods. Discussion of the limitations and impact on conclusions of missing IPD is essential.

\section{Conclusions}

Individual participant data (IPD) meta-analyses are resource demanding, time consuming, and methodologically challenging, but when conducted well, ${ }^{58}$ ideally following a registered protocol ${ }^{59}$ and adhering to the PRISMA-IPD guidance, ${ }^{57}$ can provide more detailed and potentially more reliable results than a meta-analysis of aggregate data. Meta-analysts must consider the appropriateness of an IPD analysis and document the potential biases introduced by missing such data. Only one in four published IPD meta-analyses have had access to all IPD; we hope that this proportion will increase in future years with the growing awareness of data sharing and transparency in the pharmaceutical industry and beyond. ${ }^{22-24} 26-28$ However, the research community must ensure that procedures to access IPD do not become over-burdensome, over-costly, and prohibitive, and that common sense and responsible risk proportionate approaches should be used. ${ }^{2327}$

Contributors: SJN, AGM, and CTS conceived and designed the research question. SIN, BD, SR, and LW extracted data. SIN analysed data. SJN and CTS interpreted the results. SJN wrote the manuscript under the supervision of AGM and CTS. All authors had full access to all of the data (including statistical reports and tables) in the study and can take responsibility for the integrity of the data and the accuracy of the data analysis. SJN is the guarantor.

Funding: SJN was funded by the National Institute for Health Research (NIHR) programme grants for applied research programme (grant No RP-PG-0606-1062) and by the University of Liverpool. BD, SR, and LW were funded by NIHR research methods fellowships. These funding sources had no role in the study design; in the collection, analysis, and interpretation of data; in the writing of the report; and in the decision to submit the article for publication. No other source of funding was provided to the authors or the study.

Competing interests: All authors have completed the ICMJE uniform disclosure form at www.icmje.org/coi_disclosure.pdf and declare: no support from any organisation for the submitted work; no financial relationships with any organisations that might have an interest in the submitted work in the previous three years; no other relationships or activities that could appear to have influenced the submitted work.

Ethical approval: Not required.

Data sharing: Data underlying tables and figures are available on request from the corresponding author.

Transparency: The lead author (SJN) affirms that the manuscript is an honest, accurate, and transparent account of the study being reported; that no important aspects of the study have been omitted; and that any discrepancies from the study as planned (and, if relevant, registered) have been explained.

This is an Open Access article distributed in accordance with the terms of the Creative Commons Attribution (CC BY 4.0) license, which permits others to distribute, remix, adapt and build upon this work, for commercial use, provided the original work is properly cited. See: http://creativecommons.org/licenses/by/4.0/.

1 Burns PB, Rohrich RJ, Chung KC. The levels of evidence and their role in evidence-based medicine. Plast Reconstr Surg 2011;128:305-10. doi:10.1097/PRS.0b013e318219c171.

2 Lambert PC, Sutton AJ, Abrams KR, Jones DR. A comparison of summary patient-level covariates in meta-regression with individual patient data meta-analysis. J Clin Epidemiol 2002;55:86-94. doi:10.1016/S0895-4356(01)00414-0.

3 Sutton AJ, Duval SJ, Tweedie RL, Abrams KR, Jones DR. Empirical assessment of effect of publication bias on meta-analyses. BMJ 2000;320:1574-7. doi:10.1136/bmj.320.7249.1574

4 Kirkham JJ, Dwan KM, Altman DG, et al. The impact of outcome reporting bias in randomised controlled trials on a cohort of systematic reviews. BMJ 2010;340:c365. doi:10.1136/bmj.c365.
5 Stewart LA, Tierney JF. To IPD or not to IPD? Advantages and disadvantages of systematic reviews using individual patient data. Eval Health Prof 2002:25:76-97. doi:10.1177/0163278702025001006.

6 Stewart LA, Clarke MJ. Cochrane Working Group. Practical methodology of meta-analyses (overviews) using updated individual patient data. Stat Med 1995;14:2057-79. doi:10.1002/ sim.4780141902.

7 Stewart LA, Parmar MK. Meta-analysis of the literature or of individual patient data: is there a difference?Lancet 1993;341:418-22. doi:10.1016/0140-6736(93)93004-K.

8 Tudur Smith C, Marcucci M, Nolan SJ, et al. Individual participant data meta-analyses compared with meta-analyses based on aggregate data. Cochrane Database Syst Rev 2016;9:MR000007. doi:10.1002/14651858.MR000007.pub3.

9 Simmonds M, Stewart G, Stewart L. A decade of individual participant data meta-analyses: A review of current practice. Contemp Clin Trials 2015:45(PtA):76-83. doi:10.1016/j.cct.2015.06.012.

10 Riley RD, Lambert PC, Abo-Zaid G. Meta-analysis of individual participant data: rationale, conduct, and reporting. BMJ 2010:340:c221. doi:10.1136/bmi.c221.

11 Huang Y, Mao C, Yuan J, et al. Distribution and epidemiological characteristics of published individual patient data meta-analyses. PLoS One 2014;9:e100151. doi:10.1371/journal.pone.0100151.

12 Tierney JF, Pignon JP, Gueffyier F, et al. Cochrane IPD Meta-analysis Methods Group. How individual participant data meta-analyses have influenced trial design, conduct, and analysis. J Clin Epidemiol 2015:68:1325-35. doi:10.1016/j.jclinepi.2015.05.024.

13 Vale CL, Rydzewska LH, Rovers MM, Emberson JR, Gueyffier F, Stewart LA. Cochrane IPD Meta-analysis Methods Group. Uptake of systemati reviews and meta-analyses based on individual participant data in clinical practice guidelines: descriptive study. BMJ 2015;350:h1088. doi:10.1136/bmj.h1088.

14 Riley RD, Simmonds MC, Look MP. Evidence synthesis combining individual patient data and aggregate data: a systematic review identified current practice and possible methods. J Clin Epidemiol 2007;60:431-9. doi:10.1016/j.jclinepi.2006.09.009.

15 Ahmed I, Sutton AJ, Riley RD. Assessment of publication bias, selection bias, and unavailable data in meta-analyses using individual participant data: a database survey. BMJ 2012;344:d7762. doi:10.1136/bmj.d7762

16 Jaspers GJ, Degraeuwe PL. A failed attempt to conduct an individual patient data meta-analysis. Syst Rev 2014;3:97. doi:10.1186/2046-4053-3-97.

17 Hróbjartsson A. Why did it take 19 months to retrieve clinical trial data from a non-profit organisation?BMJ 2013;347:f6927. doi:10.1136/bmj. f6927.

18 Savage CJ, Vickers AJ. Empirical study of data sharing by authors publishing in PLoS journals. PLoS One 2009;4:e7078. doi:10.1371/ journal.pone.0007078.

19 Vickers AJ. Whose data set is it anyway? Sharing raw data from randomized trials. Trials 2006;7:15. doi:10.1186/1745-6215-7-15.

20 Simmonds MC, Higgins JP, Stewart LA, Tierney JF, Clarke MJ, Thompson SG. Meta-analysis of individual patient data from randomized trials: a review of methods used in practice. Clin Trials 2005;2:209-17. doi:10.1191/1740774505cn087oa.

21 Rathi VK, Strait KM, Gross CP, et al. Predictors of clinical trial data sharing: exploratory analysis of a cross-sectional survey. Trials 2014:15:384 doi:10.1186/1745-6215-15-384.

22 Rathi V, Dzara K, Gross CP, et al. Sharing of clinical trial data among trialists: a cross sectional survey. BMJ 2012;345:e7570. doi:10.1136/ bmj.e7570.

23 Institute of Medicine (IOM) Committee on Strategies for Responsible Sharing of Clinical Trial Data. Sharing Clinical Trial Data: Maximizing Benefits. Minimizing Risk, 2015.

24 European Medicines Agency. European Medicines Agency policy on publication of clinical data for medicinal products for human use (POLICY/0070). 2014

25 Taichman DB, Backus J, Baethge C, et al. Sharing Clinical Trial Data--A Proposal from the International Committee of Medical Journal Editors. N Engl J Med 2016;374:384-6. doi:10.1056/NEJMe1515172.

26 Zarin DA. Participant-level data and the new frontier in trial transparency. N Engl J Med 2013;369:468-9. doi:10.1056/ NEJMe1307268.

27 Tudur Smith C, Hopkins C, Sydes MR, et al. How should individual participant data (IPD) from publicly funded clinical trials be shared?BMC Med 2015;13:298. doi:10.1186/s12916-015-0532-z.

28 Nisen P, Rockhold F. Access to patient-level data from GlaxoSmithKline clinical trials. N Engl J Med 2013;369:475-8. doi:10.1056/ NEJMsr1302541.

29 https://clinicalstudydatarequest.com/ (Accessed 20/04/2016) [Internet]

30 Geifman N, Bollyky J, Bhattacharya S, Butte AJ. Opening clinical trial data: are the voluntary data-sharing portals enough?BMC Med 2015;13:280. doi:10.1186/s12916-015-0525-y.

31 Strom BL, Buyse M, Hughes J, Knoppers BM. Data sharing, year 1 --access to data from industry-sponsored clinical trials. N Engl J Med 2014:371:2052-4. doi:10.1056/NEJMp1411794. 
32 Homer P, Gross M. Data Unleashed: Cooperation Among Competitors. Pharmaceutical Executive. 2016 April 272016.

33 Mayo-Wilson E, Doshi P, Dickersin K. Are manufacturers sharing data as promised?BMJ 2015;351:h4169. doi:10.1136/bmj.h4169.

34 Marson AG, Williamson PR, Hutton JL, Clough HE, Chadwick DW. Carbamazepine versus valproate monotherapy for epilepsy. Cochrane Database Syst Rev 2000;(3):CD001030.

35 Nolan SJ, Sudell M, Tudur Smith C, Marson AG. Topiramate versus carbamazepine monotherapy for epilepsy: an individual participant data review. Cochrane Database Syst Rev 2016:12:CD012065.

36 Nolan SJ, Marson AG, Weston J, Tudur Smith C. Phenytoin versus valproate monotherapy for partial onset seizures and generalised onset tonic-clonic seizures: an individual participant data review. Cochrane Database Syst Rev 2016;4:CD001769.

37 Nolan SJ, Tudur Smith C, Pulman J, Marson AG. Phenobarbitone versus phenytoin monotherapy for partial onset seizures and generalised onset tonic-clonic seizures. Cochrane Database Syst Rev 2013;1:CD002217.

38 Nolan SJ, Muller M, Tudur Smith C, Marson AG. Oxcarbazepine versus phenytoin monotherapy for epilepsy. Cochrane Database Syst Rev 2013;5:CD003615.

39 Nolan SJ, Tudur Smith C, Weston J. A.G. M. Lamotrigine versus carbamazepine monotherapy for epilepsy. Cochrane Database Syst Rev 2016;(11):CD001031.

40 Nolan SJ, Marson AG, Weston J, Tudur Smith C. Carbamazepine versus phenytoin monotherapy for epilepsy: an individual participant data review. Cochrane Database Syst Rev 2015;8:CD001911.

41 Nolan SJ, Marson AG, Weston J, Tudur Smith C. Carbamazepine versus phenobarbitone monotherapy for epilepsy: an individual participant data review. Cochrane Database Syst Rev 2015;7:CD001904.

42 Nolan SJ, Sudell M, Weston J, Tudur Smith C, Marson AG. Antiepileptic drug monotherapy for epilepsy: a network meta-analysis. Cochrane Database Syst Rev 2014;(12):CD011412.

43 International League Against Epilepsy. Considerations on designing clinical trials to evaluate the place of new antiepileptic drugs in the treatment of newly diagnosed and chronic patients with epilepsy. Epilepsia 1998;39:799-803. doi:10.1111/j.1528-1157.1998.tb01167.x.

44 Williamson PR, Marson AG, Tudur C, Hutton IL, Chadwick D. Individual patient data meta-analysis of randomized anti-epileptic drug monotherapy trials. J Eval Clin Pract 2000;6:205-14. doi:10.1046/j.1365-2753.2000.00236x.

45 Tudur Smith C, Marson AG, Chadwick DW, Williamson PR. Multiple treatment comparisons in epilepsy monotherapy trials. Trials 2007;8:34. doi:10.1186/1745-6215-8-34.

46 Tudur Smith C, Marson AG, Williamson PR. Carbamazepine versus phenobarbitone monotherapy for epilepsy[Review] [21 refs]. Cochrane Database Syst Rev 2003;(1):CD001904.

47 Tudur Smith C, Marson AG, Clough HE, Williamson PR. Carbamazepine versus phenytoin monotherapy for epilepsy[Review] [47 refs]. Cochrane Database Syst Rev 2002;(2):CD001911.

48 Tudur Smith C, Marson AG, Williamson PR. Phenytoin versus valproate monotherapy for partial onset seizures and generalized onset tonic-clonic seizures[Review] [40 refs]. Cochrane Database Syst Rev 2001;(4):CD001769.
49 Taylor S, Tudur Smith C, Williamson PR, Marson AG. Phenobarbitone versus phenytoin monotherapy for partial onset seizures and generalized onset tonic-clonic seizures[Review] [16 refs]. Cochrane Database Syst Rev 2001;(4):CD002217.

50 Muller M, Marson AG, Williamson PR. Oxcarbazepine versus phenytoin monotherapy for epilepsy[Review] [20 refs]. Cochrane Database Syst Rev 2006; (2):CD003615.

51 Gamble CL, Williamson PR, Marson AG. Lamotrigine versus carbamazepine monotherapy for epilepsy. Cochrane Database Syst Rev 2006:(1):CD001031.

52 Marson AG, Al-Kharusi AM, Alwaidh M, et al. SANAD Study group. The SANAD study of effectiveness of carbamazepine, gabapentin, lamotrigine, oxcarbazepine, or topiramate for treatment of partial epilepsy: an unblinded randomised controlled trial. Lancet 2007:369:1000-15 doi:10.1016/S0140-6736(07)60460-7.

53 Marson AG, Al-Kharusi AM, Alwaidh M, et al. SANAD Study group. The SANAD study of effectiveness of valproate, lamotrigine, or topiramate for generalised and unclassifiable epilepsy: an unblinded randomised controlled trial. Lancet 2007;369:1016-26. doi:10.1016/ S0140-6736(07)60461-9.

54 Debray TP, Moons KG, van Valkenhoef G, et al. GetReal Methods Review Group. Get real in individual participant data (IPD) meta-analysis: a review of the methodology. Res Synth Methods 2015;6:293-309. doi:10.1002/jrsm.1160

55 European Union Clinical Trials Directive. Directive 2001/20/EC of the European Parliament and of the Council of 4 April 2001 on the approximation of the laws, regulations and administrative provisions of the Member States relating to the implementation of good clinical practice in the conduct of clinical trials on medicinal products for human use. 2001.

56 Zanchetti A, Mancia G. Searching for information from unreported trials--amnesty for the past and prospective meta-analyses for the future. J Hypertens 1998;16:125. doi:10.1097/00004872-199816020-00001.

57 Stewart LA, Clarke M, Rovers M, et al. PRISMA-IPD Development Group. Preferred Reporting Items for Systematic Review and Meta-Analyses of individual participant data: the PRISMA-IPD Statement. JAMA 2015;313:1657-65. doi:10.1001/jama.2015.3656.

58 Tierney JF, Vale C, Riley R, et al. Individual Participant Data (IPD) Meta-analyses of Randomised Controlled Trials: Guidance on Their Use. PLOS Med 2015;12:e1001855. doi:10.1371/journal.pmed.1001855.

59 Booth A, Clarke M, Dooley G, et al. The nuts and bolts of PROSPERO: an international prospective register of systematic reviews. Syst Rev 2012;1:2. doi:10.1186/2046-4053-1-2

Appendix 1: Details of search strategies

Appendix 2: Data extraction form

Appendix 3: Statistical analysis

Appendix 4: Reference details of 1278 eligible articles describing 1280 IPD meta-analyses

Appendix 5: Outcome of IPD requests Appendix figure: Characteristics of systematic IPD meta-analyses and proportion of IPD provided 